Comment les ports de commerce devinrent-ils des nœuds de communication? Les leçons de l'histoire française

How did commercial harbours become communication nodes?

\title{
Bruno Marnot
}

\section{OpenEdition}

\section{Journals}

Édition électronique

URL : https://journals.openedition.org/rhcf/1520

DOI : 10.4000/rhcf.1520

Éditeur

Rails \& histoire

Édition imprimée

Date de publication : 1 septembre 2012

Pagination : 9-26

ISSN : 0996-9403

\section{Référence électronique}

Bruno Marnot, «Comment les ports de commerce devinrent-ils des nœuds de communication ? Les leçons de l'histoire française ", Revue d'histoire des chemins de fer [En ligne], 42-43 | 2012, mis en ligne le 14 novembre 2014, consulté le 22 avril 2022. URL : http://journals.openedition.org/rhcf/1520 ; DOI : https://doi.org/10.4000/rhcf.1520 


\section{Comment les ports de commerce devinrent- ils des nœuds de communication? Les leçons de I'histoire française}

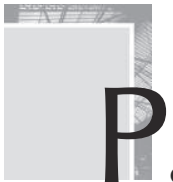

our le géographe, le port est d'abord une « aire de mise en contact entre deux domaines de circulation ». Cette réalité suppose des moyens et des processus de mise en relation de ces deux domaines de circulation qui contrôlent, chacun de leur côté, des espaces plus ou moins étendus. Ces moyens et processus de mise en relation s'appellent des nœuds de communication. Si l'on raisonne en termes de trafic, ces nœuds s'apparentent à des points de concentration et de redistribution des marchandises et des hommes. Cela signifie que les ports se sont intégrés à la logique des réseaux, dont ils sont en quelque sorte devenus des points névralgiques.

Cette fonction a impliqué une nouvelle conception de la desserte portuaire à partir du XIx ${ }^{\mathrm{e}}$ siècle. Les ports sont, en effet, à la fois rupture de charge et nœuds de communication : la première notion évoque la discontinuité, la deuxième la continuité. Mais, depuis le $\mathrm{XIX}^{\mathrm{e}}$ siècle, la deuxième notion tend toujours plus à faire oublier la première. Pour les ports, devenir des nouds de communication signifiait d'être capable d'assurer la meilleure transition possible entre transports maritimes et terrestres. Les ports sont ainsi probablement devenus les premières plate-formes multimodales et, par conséquent, les premiers espaces à devoir penser et gérer l'intermodalité des transports. 
Le principe du nœud de communication est intimement lié à l'explosion du commerce maritime mondial et au développement de l'activité de transit ${ }^{1}$ terrestre tout au long du XIX ${ }^{e}$ siècle. Si les ports sont devenus des nœuds de communication, c'était dans l'optique de mieux contrôler à la fois leur avantpays et leur arrière-pays, en raison d'une concurrence acharnée pour la captation des divers trafics. Cette condition supposait d'obtenir une interface avant-pays/arrière-pays optimale, qui elle même reposait sur des contraintes techniques, commerciales et tarifaires d'un nouvel ordre :

- Sur le plan technique, l'objectif d'offrir une logistique optimale - c'està-dire la recherche des meilleures conditions d'acheminement et de réexpédition des marchandises, tenant compte des coûts, des délais et de la qualité des services - devint la règle absolue.

- Côté maritime : pour attirer les armements, il faut des trafics massifs, susceptibles de fournir, avec régularité et durablement, des cargaisons abondantes et fructueuses.

- Côté terrestre : pour attirer les trafics, nécessité de moyens de transbordement et d'acheminement vers les centres de consommation, rapides, efficaces, au moindre coût.

Au total, la nécessité d'assurer la meilleure interface terre/mer a été sans doute l'un des plus puissants facteurs de modernisation des principaux ports de commerce français au $\mathrm{XIX}^{\mathrm{e}}$ siècle. Cette logique a eu des effets profondément sélectifs et discriminants : seuls les quelques ports qui occupaient déjà le sommet de la hiérarchie ont pu prétendre devenir des nœuds de communication à l'échelle régionale, nationale ou internationale.

Notre propos consistera à s'interroger sur les prétentions des ports français à s'ériger en nœuds de communication à une échelle non pas seulement régionale ou même nationale, mais surtout européenne. Ceci revient à s'interroger sur la lecture que les ports ont faite de l'évolution des principaux flux maritimes et terrestres et des moyens qu'ils ont déployés pour tenter de les capter. Trois facteurs, qui furent selon les cas perçus comme des atouts ou des contraintes, ont constamment influencé la transformation des ports en nœuds de communication : l'espace, les opérateurs de transport, les institutions politiques.

1- Traversée de la marchandise dans un espace portuaire, à destination ou en provenance de l'arrière-pays. 


\section{La situation faussement avantageuse des ports français à l'échelle européenne}

Le problème de l'interface avant-pays/arrière-pays s'est posé de façon originale dans le cas de la France qui disposait a priori d'une situation avantageuse, puisqu'elle pouvait se présenter comme une véritable tête de pont du continent européen, ouverte sur les deux espaces maritimes commerciaux alors parmi les plus dynamiques du monde, à savoir l'océan Atlantique et la Méditerranée.

Les élites négociantes des différentes villes maritimes françaises étaient bien conscientes de cet atout potentiel et chaque port important a tenté de faire valoir auprès des pouvoirs publics l'excellence de sa situation par rapport à ses concurrents régionaux et nationaux, dans le but de jouir d'un traitement de faveur en matière d'équipement. Le Havre se présentait ainsi comme la " porte océane " par excellence : porte d'entrée de Paris, de l'est de la France, de l'axe vers la Méditerranée, depuis que l'axe rhodanien, comme l'écrivait Louis Girard, avait pris une valeur mondiale. À l'autre extrémité, Marseille, le plus grand port français, rêvait d'une reconquête, par l'arrière-pays continental, de marchés qui dépendaient autrefois de l'entrepôt maritime.

«Le temps n'est pas éloigné, écrivait un éminent observateur local en 1842, où, grâce aux perfectionnements de nos fleuves et de nos chemins, les marchandises pourront aller, d'un bout à l'autre de la France, à moindre frais qu'il n'en faut aujourd'hui pour aller par mer d'un port de la Méditerranée à un port de l'Océan. Marseille sera alors le port de toute l'Europe. Le transit pour l'Angleterre et l'Allemagne, dont elle fut si longtemps en possession, qui fut abandonné par suite des progrès de la navigation, lui reviendra par suite des progrès de la navigation intérieure ${ }^{2}$."

Brest, dont l'activité commerciale était pourtant embryonnaire, excipait de sa position exceptionnelle de "finisterre " de l'Europe pour se présenter comme le futur "Faubourg de Paris " ou la " Marseille de l'Océan », en devenant à la fois port de transit, marché des denrées d'outre-mer et port d'armement $^{3}$ (fig. 1). Même enjeu à Nantes qui rêvait aussi de devenir la porte d'entrée maritime de Paris et de l'Europe centrale. D’où la nécessité de rompre l'isolement par l'élargissement de l'arrière-pays vers l'intérieur et, par conséquent, par l'établissement d'une grande voie transversale vers la Suisse et l'Allemagne. Dans ce contexte, l'ensemble Nantes - Saint-Nazaire était appelé à devenir le terminus du grand axe Suisse-Océan. Saint-Nazaire serait une succursale de

2- Jules Julliany, Essai sur le commerce de Marseille, Marseille, 1842 ( $2^{\mathrm{e}}$ éd.), cité par RONCAYOLO, 1990, p. 47.

3- CLOÎTRE-QUÉRÉ, 1992, p. 176. 
Nantes comme Bremerhaven de Brême. Pour d'autres observateurs, il s'agissait de faire de Nantes le "Liverpool de l'Ouest ", modèle également caressé par La Rochelle... Les grands ports européens servirent de références constantes aux ambitions des établissements français.

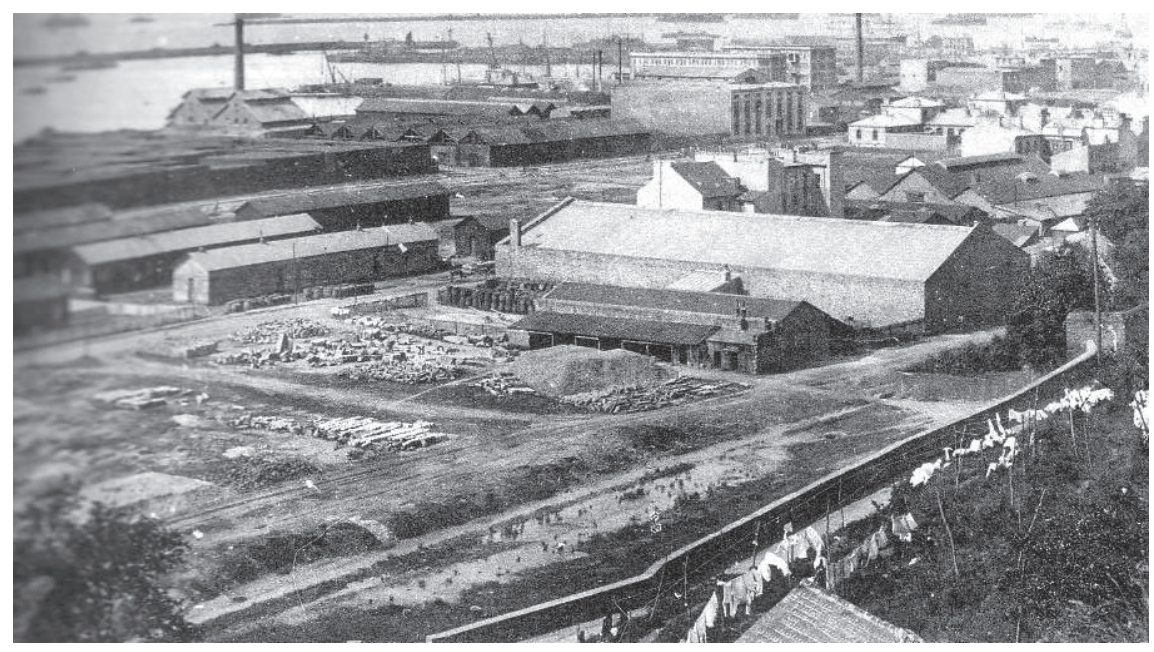

Figure 1. Brest : éphémère escale transatlantique et illusoire "Marseille de I'Océan". Carte postale, coll. et cl. auteur.

Trois constats ressortent de ces projections. Le premier repose sur le fait que la croissance portuaire implique désormais que l'hinterland doit dépasser les limites ancestrales de "l'arrière-pays fondamental». Cet hinterland plus profond est désigné, par les géographes fonctionnalistes, par l'expression de "marges de compétition ». En deuxième lieu, il s'avère que le nouvel horizon terrestre que les plus grands ports français souhaitent atteindre s'incarne dans le cour économique et démographique de l'Europe, depuis l'Italie du Nord jusqu'à l'Allemagne rhénane. Enfin, troisième constat, seul un système de transports terrestre efficace peut permettre d'atteindre et de capter les trafics de ce nouvel horizon terrestre.

Au sein des grands établissements français, le débat des années 1850-1860 a tourné autour de l'articulation entre le port et les voies de communication terrestres, même si le choix n'était pas toujours fait entre voie navigable et chemin de fer. En arrière-plan, ce fut bien la mutation de l'hinterland des ports qui fut envisagée par les différents acteurs locaux. Comme l'écrit l'historienne 
de Nantes, A. Vauthier-Vézier, " une autre logique de l'espace intérieur se met en place qui situe le port comme point terminal d'un réseau de voies ferrées $~^{4}$. Ces spéculations sur l'avenir des ports renvoient bien à cette vision assez frappante jusqu'aux années 1860, selon M. Roncayolo, d'un «territoire en mouvement ", d'un " champ des possibles [...] visible dans les évolutions amorcées et dans l'imaginaire déployé $»^{5}$.

Les élites portuaires ont ainsi pris conscience du fait que la dilatation de leur arrière-pays passait par la structuration des réseaux de communications et par l'animation des flux qui partaient du port ou y aboutissaient. Audelà des réseaux de communication, l'industrialisation fut également perçue comme l'un des plus puissants agents structurants des espaces terrestres par ses effets d'agglomération démographique (urbanisation) et ses incidences sur le système des transports. Aussi la plupart des élites portuaires mesurèrentelles pleinement les incidences de la nouvelle donne économique et spatiale qui était à l'œuvre, à savoir le déplacement d'activité et de prospérité vers le nord et l'est de la France, et plus largement vers ce nord-ouest européen avec lequel il fallait impérativement entrer en contact sous peine de connaître un irrémédiable déclassement économique. La condition sine qua non consistait bien à intégrer le port dans une chaîne de transports multimodale dont il devait devenir l'un des points d'appui stratégiques.

\section{Les opérateurs de transport et la logique de la rentabilité}

Cette géographie repensée par les élites portuaires ne coïncidait pas forcément avec la vision des firmes de transport maritime et terrestre qui, évoluant elles-mêmes dans un univers concurrentiel, cherchèrent à mettre en place une autre géographie repensée à partir du critère tarifaire.

La théorie fonctionnaliste nous a enseigné que, au-delà de la question des infrastructures, l'un des principaux moyens mis en œuvre par les ports pour élargir leur arrière-pays dans les "marges de compétition " consiste à abaisser "l'équation globale des coûts de circulation ». Celle-ci correspond au parcours global de la marchandise le long d'une chaîne de transport complète qui comprend deux triptyques portuaires ayant en commun le même avant-pays (fig. 2).

4- VAUTHIER-VEZIER, 1997, p. 438.

5- RONCAYOLO, 2000, p. 423. 
Figure 2. L'équation des coûts de circulation appliquée aux ports ${ }^{1}$

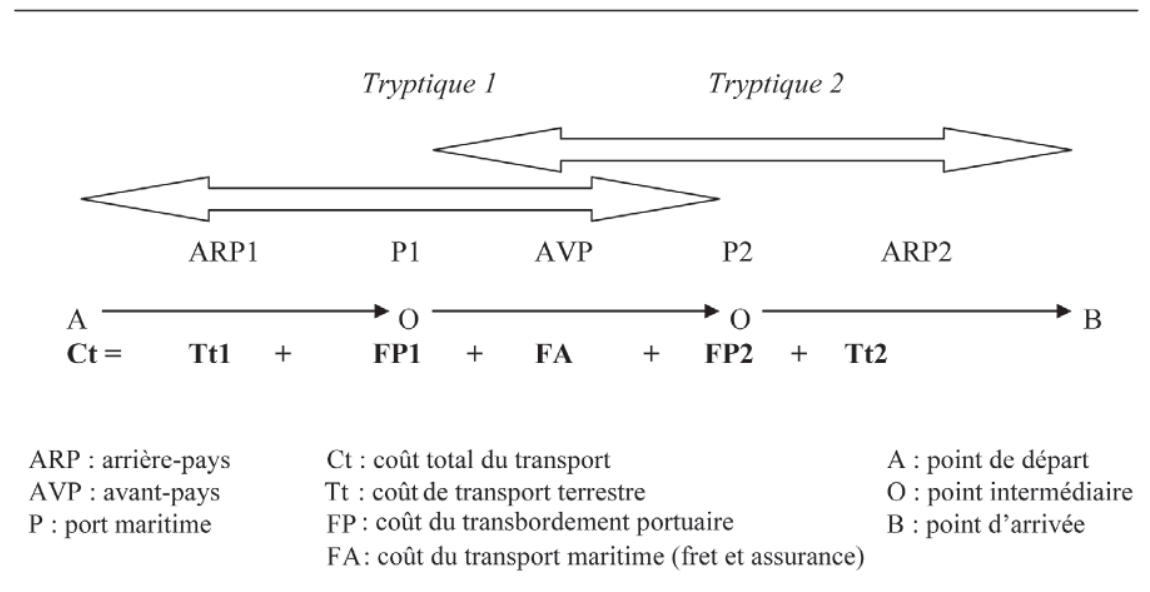

${ }^{1}$ Source : d'après André Vigarié, «Les ports maritimes et leur environnement humain et économique », in A. Gamblin (dir.). Les Littoraux, espaces de vie, Paris, SEDES, 1998, p. 72.

L'équation des coûts de circulation est la modélisation simple de ce que doit payer l'expéditeur de la marchandise. Le port ne peut directement agir que sur un des seuls membres de l'équation, c'est-à-dire l'abaissement de FP1 s'il se trouve dans le triptyque 1 ou de FP2 s'il se trouve dans le triptyque 2. Les autres membres de l'équation, à savoir le prix du transport terrestre $(\mathrm{Tt})$ et le fret maritime (FA), dépendent des décisions des compagnies. Les ports ont ainsi cherché, d'un côté, à attirer les armateurs par une réduction du coût des escales, de l'autre, à obtenir des tarifications préférentielles de la part des opérateurs terrestres, plus particulièrement des compagnies de chemins de fer qui, par exemple dans les cas des ports du Sud-Ouest, étaient les seuls réseaux à assurer la connexion avec le débouché parisien tant recherché.

Les opérateurs de transport ont évidemment joué de cette concurrence portuaire pour guider ou attirer les trafics. À l'instar des compagnies de chemins de fer, les grandes compagnies d'armement ont, au XIx ${ }^{e}$ siècle, structuré les espaces maritimes avec la constitution de lignes dont le principe était de générer la formation d'un important trafic de passagers et de marchandises. Les lignes reliaient des destinations dont la desserte était assurée à fréquence régulière. L'organisation des lignes explique la lutte acharnée que se sont livrée les ports pour obtenir la tête ou l'escale, à tel point que l'accueil des paquebots 
est devenu, dès le mitan du XIX ${ }^{e}$ siècle, un puissant ferment de la modernisation des structures portuaires (fig. 3).

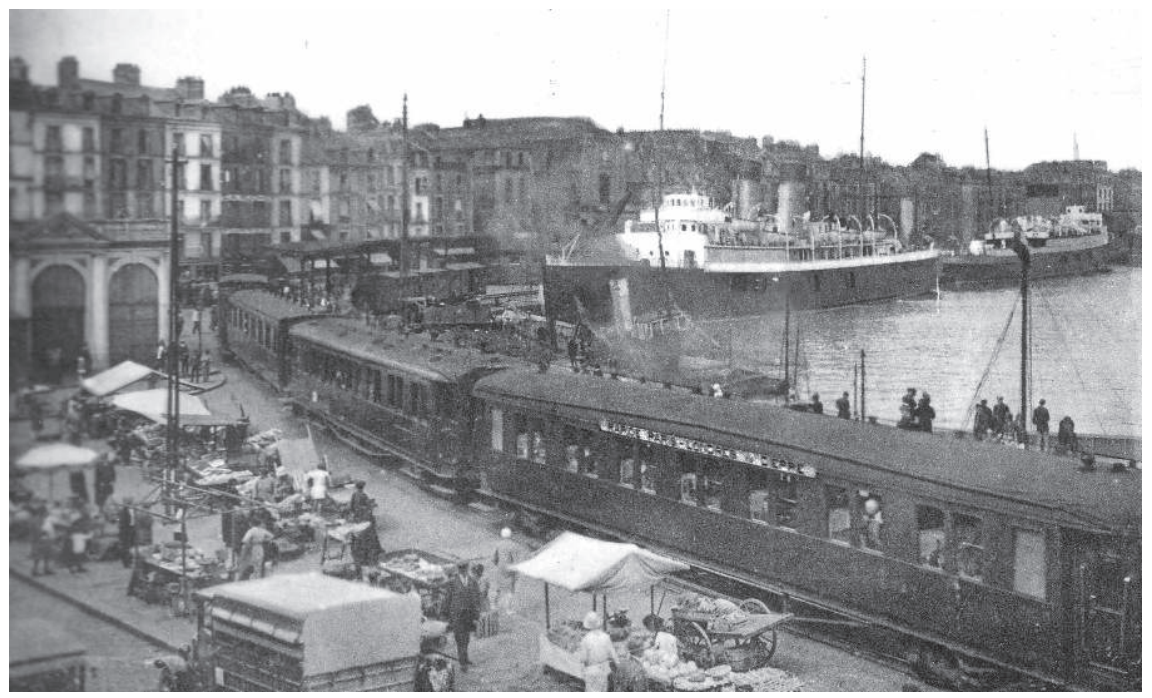

Figure 3. Un exemple d'intermodalité : le départ du train maritime à Dieppe. Carte postale, coll. et cl. auteur.

Mais si les historiens ont souvent mis l'accent, à juste titre, sur les transformations structurelles de l'économie maritime comme facteur majeur de la modernisation portuaire, ils ont eu tendance à sous-estimer, me semble-t-il, la problématique continentale sans laquelle ne serait pas totalement compréhensible l'évolution, en qualité comme en quantité, des flux maritimes. L'interconnexion avec les réseaux ferroviaires a joué un rôle tout aussi structurant du nouvel espace portuaire. Pour les acteurs locaux impliqués dans la modernisation de leur outil, on ne pouvait pas penser l'interface maritime sans son prolongement terrestre.

On comprend dès lors l'empressement des villes portuaires - une fois les réticences locales surmontées dans quelques cas - à réclamer une connexion avec la voie de fer, surtout quand elles étaient dépourvues de tout réseau navigable. Quelques années de décalage dans l'établissement d'une desserte ferroviaire étaient déterminantes pour capter ou perdre les nouveaux marchés situés au-delà de l'horizon terrestre traditionnel. Le réseau en étoile institué par la loi de 1842 favorisa les liaisons entre la capitale et les grands ports, mais la chronologie des connexions se caractérisa par des écarts importants. Ainsi 
Rouen fut relié à Paris dès 1843. Le Havre obtint sa liaison quatre ans plus tard, Dunkerque, Calais et Boulogne en 1848. En 1851, Nantes était desservie par une ligne qui communiquait avec Tours et, au-delà, avec Paris, tandis que Bordeaux dut attendre 1853 pour que la liaison sans rupture de charge avec la capitale fût réalisée. La Rochelle, qui était d'un intérêt secondaire pour la Compagnie de l'Orléans dut patienter jusqu'en 1857. À Marseille, l'arrivée du chemin de fer se produisit en 1848, depuis Avignon, avant que ne fût exécutée la connexion avec Lyon en 1853, date à laquelle la ligne rencontra celle de Paris. Entre Rouen et le port phocéen, un décalage de dix années s'était produit en ce qui concerne la connexion directe avec Paris, qui constituait le plus grand marché français et par conséquent l'objet de toutes les convoitises (fig. 4). Ces écarts s'expliquaient autant par la différence des distances à couvrir que par la nécessité de surmonter des problèmes techniques, financiers ou institutionnels, comme le regroupement des compagnies.

À leur tour, les compagnies de chemins de fer ont usé de stratégies tarifaires complexes qui ont, selon les types de trafics et les directions, fait varier l'attractivité des ports. Ces stratégies ont joué un rôle particulièrement décisif en ce qui concerne le transit international, où l'espace à couvrir pour les opérateurs terrestres dépassait le cadre des frontières nationales, ce qui représentait un fret particulièrement recherché en raison de la longueur des distances à parcourir. Ainsi l'enjeu de la lutte particulièrement âpre entre les grands ports français comme Le Havre, Dunkerque et leurs rivaux belge et hollandais (Anvers, Rotterdam) fut la desserte de l'hinterland rhénan, allemand et parisien. Anvers s'est imposé, à la fin du XIx ${ }^{\mathrm{e}}$ siècle, comme le principal nœud de communication des routes maritimes et terrestres de l'Europe du NordOuest, en jouant davantage sur sa situation que sur le coût de ses propres prestations. À la qualité de son interface terre-mer, qui se caractérisait par une interconnexion remarquable entre le navire et le rail, s'ajoutèrent l'excellence de la liaison terrestre jusqu’à Bâle et la compétitivité des prix pratiqués par les chemins de fer belges par rapport à leurs homologues français ${ }^{6}$. Les ports français de la région étaient donc particulièrement désavantagés du côté terrestre. Non pas qu'ils fussent forcément plus chers que leur rival belge et qu'ils fussent donc moins susceptibles d'attirer le fret maritime, mais l'interface terrestre était particulièrement négligée. Elle le fut à la fois sur les plans technique, commercial et tarifaire.

6- VERAGHTERT, 1975, p. 113 et suiv. 


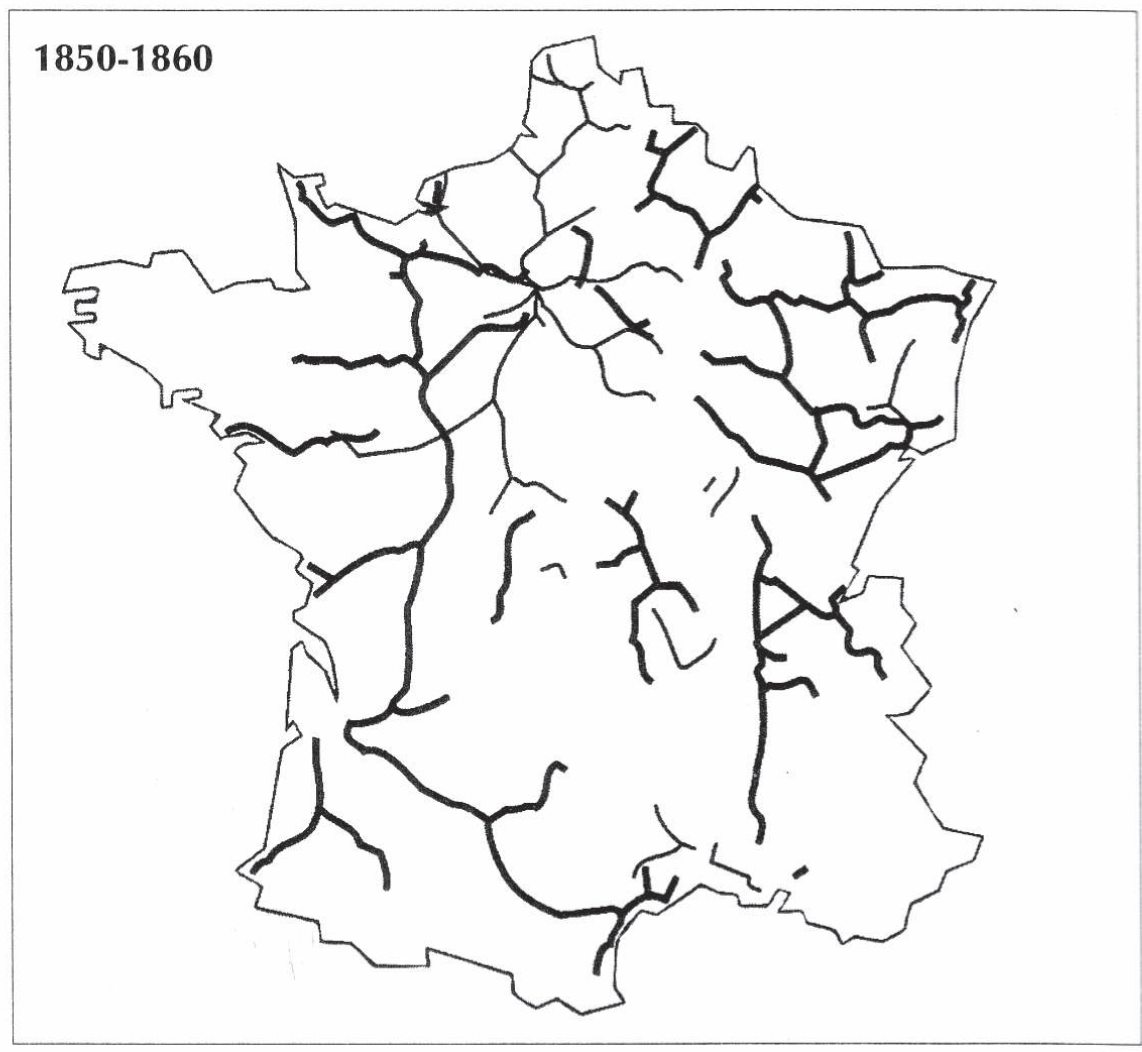

Voie ferrée ancienne

Figure 4. L'inégale connexion des ports maritimes français à Paris par voie ferrée au milieu du XIX siècle. Source : Gérard Blier, Nouvelle géographie ferroviaire de la France, t. 1, Paris, éd. La Vie du rail, 1991, p. 13. 
Le premier point pourrait être illustré par la perte progressive de l'arrièrepays rhénan par le port du Havre. Si l'on considère les seules causes du côté français, Le Havre a autant souffert d'une desserte ferroviaire insuffisante que d'une voie navigable qui profita surtout à son rival rouennais. Pourtant, à l'origine, la compagnie concessionnaire trouva un accueil favorable au Havre, qu'elle connecta à sa ligne parisienne en 1847. Comme elle avait, par ailleurs, avantage à utiliser sa voie ferrée sur tout le parcours, elle commença par s'appuyer sur ce port. Cependant, les relations se dégradèrent assez vite au cours des années suivantes, en raison des plaintes répétées des Havrais qui souffraient de l'insuffisance des relations avec l'intérieur, alors que le trafic portuaire était en plein essor. La Seine étant impraticable au niveau de l'embouchure, Le Havre ne pouvait compter que sur l'existence d'une seule ligne, ce qui était considéré comme un facteur de vulnérabilité. Aussi les élus locaux et la chambre de commerce multiplièrent-ils les requêtes pour obtenir l'ouverture d'une deuxième ligne, sur les plateaux, qui doublerait celle de la vallée. La Compagnie de l'Ouest les éconduisit régulièrement dans la mesure où elle ne voyait pas d'effet de levier quelconque qui pût produire une augmentation du trafic. Le Havre se heurta, de plus, à l'opposition de Rouen qui consentait, au même moment, d'énormes efforts pour l'amélioration du chenal maritime. Ce fut ce dernier port qui prit un avantage décisif dans une concurrence âpre et interminable pour desservir la région parisienne et une partie de l'est de la France. Outre ses efforts fructueux pour améliorer la navigation sur la Seine, le port normand se mua en véritable nœud ferroviaire au début du $\mathrm{xx}^{\mathrm{e}}$ siècle. À l'inverse, l'absence d'une seconde liaison ferroviaire au Havre gêna considérablement l'essor de ses liaisons continentales. En 1914, les tonnages transportés par chemin de fer depuis Le Havre étaient les plus faibles parmi les grands ports européens. Par ailleurs, l'avant-port de la Seine se caractérisait par l'extrême faiblesse des relations extérieures au réseau de l'Ouest. On comprend dès lors la volonté farouche des Havrais de sortir du carcan que lui imposait la compagnie en recherchant la construction d'un canal de jonction avec la Seine, mais aussi l'intense activité du cabotage et des sorties d'entrepôt par mer qui tentèrent de compenser le rétrécissement relatif de l'horizon terrestre du port ${ }^{7}$.

L'aspect commercial de l'interface terrestre fut notamment soulevé par l'inspecteur principal Marie, directeur du commerce extérieur au ministère du Commerce, dans le rapport qu'il remit à la commission d'enquête de 1882 sur la situation de grands ports français de la Manche et de la Mer

7- VIGARIÉ, 1964, p. 319-322 ; CROGUENNEC, 1999, p. 120-122. 
du Nord. Selon lui, la véritable solution consistait dans le groupage des marchandises, ainsi qu'il se pratiquait déjà en Belgique et en Allemagne. Cette proposition radicale demandait tout simplement aux compagnies de chemins de fer de rompre avec des pratiques commerciales de plusieurs décennies. L'absence du groupage évitait, en effet, le recours à l'intermédiaire, au commissionnaire, dont le travail consistait, en l'occurrence, à réunir différents types de marchandises pour effectuer des chargements complets. Cette tâche logistique ôtait aux compagnies une grande partie du travail en leur livrant des wagons entièrement chargés. Mais en France les commissionnaires furent considérés, dès la mise en place des réseaux, comme des " parasites ", pour reprendre le mot de F. Caron, tant par la haute administration que par les directions des compagnies. Cependant, le refus du groupage se traduisit par la difficulté croissante à respecter les délais de livraison et une élévation du coût du transport. Aussi Marie proposait-il, en 1882, de faire « l'essai, sur une vaste échelle, $[\mathrm{du}]$ chargement par wagon complet de 10 tonnes, à prix réduit, sans distinction de nature de marchandises, aux risques et périls de l'expéditeur ". Sa proposition buta sur l'opposition du Comité consultatif des chemins de fer qui ne dérogea pas à la doctrine originelle.

Enfin, sur le troisième point, Marie dénonçait la rigidité du système tarifaire français. Le non-sens économique des décrets de 1862 et 1864 était mis sur la sellette, à la lumière des exemples allemand et belge où "dans l'établissement des taxes, les chemins de fer [...] conservent les distances respectives des ports de mer aux points de provenance ou de destination, quelques faibles que soient d'ailleurs les différences de parcours $»^{8}$. Le système français des tarifs de transit internationaux reposait, au contraire, depuis le Second Empire, sur un principe bureaucratique et égalitaire, conçu à l'origine pour rétablir la compétitivité des compagnies ferroviaires françaises, qui souffraient des lenteurs du processus d'homologation des tarifs par rapport aux transports fluviaux et aux réseaux ferrés étrangers. Un premier décret, du 26 avril 1862, leur donna en partie satisfaction : les compagnies étaient dispensées de l'homologation et de l'affichage préalable pour les tarifs de transit, mais l'administration inclut, en contrepartie, une clause établissant l'égalité de traitement pour tous les ports d'un même littoral desservis par un même réseau. L'égalitarisme forcené de ce système fut atténué par un deuxième décret, du $1^{\text {er }}$ août 1864 , qui établissait treize groupes de ports, au sein desquels s'appliquait la règle de l'égalité. Par exemple, les ports de la Compagnie du Nord, ramassés sur une petite portion du littoral de la Manche et de la Mer du

8- Ministère des Travaux publics, 1883, p. 78. 
Nord, ne formaient qu'un seul groupe, mais ceux de l'Ouest étaient divisés en trois ensembles, de Dieppe à Caen, de Caen à Saint-Brieuc et de Saint-Brieuc à Brest ${ }^{9}$. Malgré les recommandations de Marie, qui demandait de suivre les exemples des pays voisins, le principe de l'égalité de traitement des ports face aux tarifs de transit perdura jusqu'à la veille de la Première Guerre mondiale, en dépit d'une légère révision en 1894.

Par la situation de la France, la fonction de transit pouvait aussi prendre la forme du passage transisthmique reliant la Manche à la Méditerranée. Des projets de ligne directe entre les deux mers furent évoqués très tôt dans le siècle. En février 1831, Duveyrier prônait dans l'organe des saint-simoniens Le Globe l'exécution d'une artère unique mais vitale qui joindrait Le Havre à Marseille, projet soutenu par Stéphane Flachat, puis reprit par Blum et Chevalier en 1832, ainsi que par l'ingénieur Henri Fournel en 1833. À considérer ces premières esquisses, la ligne Manche-Méditerranée était à chaque fois l'axe central du futur réseau de chemins de fer. Le projet, un temps oublié, ressurgit avec force au lendemain du Second Empire, dans le but à peine dissimulé de briser le monopole des réseaux du Nord et du PLM. Trois hommes d'affaires, Donon, Delahante et Gladstone présentèrent la liaison ferroviaire Calais-Marseille comme la possible grande ligne de transit française entre l'Angleterre et l'Orient. Leurs arguments reposait sur l'incapacité des grandes compagnies, complètement désorganisées par la guerre, à répondre aux besoins du trafic, lequel ne cessait de croître entre le Nord-Ouest de l'Europe et l'espace méditerranéen, en particulier depuis l'ouverture de Suez. Toutefois, le projet ne fut pas soutenu à l'Assemblée Nationale par le rapporteur de la proposition de loi, l'ingénieur des Ponts et Chaussées Louis-Ernest Cézanne. Ce dernier, qui avait passé une grande partie de sa carrière dans les chemins de fer, suivit plutôt les avis des compagnies du Nord et du PLM qui firent valoir que le trafic de transit était insignifiant le long de cet axe. De l'avis même du Conseil général des Ponts et Chaussées, les encombrements qu'on avait pu constater résultaient plutôt de l'insuffisance des installations dans la plupart des gares. La solution la plus sage consistait donc à améliorer le réseau existant plutôt que d'en créer un de toutes pièces qui conduirait à une concurrence ruineuse $^{10}$. L'alliance objective d'une puissante administration technique et de deux non moins puissantes compagnies enterrèrent donc le projet de liaison directe Calais-Marseille.

9- PICARD, 1918, p. 847-848 ; COLSON, 1898-1903, p. 135.

10- Sur le projet Calais-Marseille voir PICARD, 1884, p. 98-110 ; CARON, 1997, p. 427-428 ; MARNOT, 2000, p. 46-47. 


\section{Les institutions politiques : la défaillance de l'État}

L'État français, qui était propriétaire des ports, a pris tardivement conscience de la nécessité de mieux " nouer " les établissements portuaires aux grands axes maritimes et terrestres. Outre un état d'esprit continentaliste dominant, tout au long du XIX siècle, chez la plupart des élus et des dirigeants politiques français, l'un des obstacles majeurs à cette prise de conscience résida dans l'éparpillement des crédits qu'un système d'avances et de subsides effectués par les collectivités locales (chambres de commerce, municipalités, conseils généraux) encouragea fortement. La philosophie budgétaire des pouvoirs publics français en ce domaine a été guidée, de façon chronique, par un raisonnement limité à l'échelle nationale et par un souci de justice redistributive entre plusieurs dizaines d'établissements commerciaux, le plus souvent d'importance médiocre, disséminés le long de deux longues façades littorales.

Le plan Freycinet de 1879 illustre parfaitement les ambitions étriquées d'un projet d'interconnexion des ports aux voies navigables et aux artères ferroviaires, qui était incapable de prendre sérieusement en considération le dynamisme croissant des autres ports européens et, par conséquent, la menace qu'ils faisaient peser sur la compétitivité des grands établissements français. Â cet égard, le plus important port de l'hexagone, Marseille, se retrouva en position délicate à l'aube des années 1880 . Au lieu de recueillir les bénéfices de l'ouverture du canal de Suez, la cité phocéenne ressentit de plus en plus la menace de Gênes, en particulier depuis l'inauguration du tunnel du SaintGothard en 1882. Le transit entre l'Angleterre et l'Orient échappait de plus en plus à Marseille, soit au profit de la liaison maritime directe avec les puissants organismes de la mer du Nord, soit au profit de l'axe scaldéo-rhénan via Gênes. Les compagnies de chemins de fer françaises furent par ailleurs réservées quant aux possibilités de développer un trafic de transit vers l'Italie, la Suisse et l'Allemagne. L'ouverture, en 1871, du tunnel du Mont-Cenis, à l'extrémité de la vallée de la Maurienne, offrait une solution, mais les courants commerciaux butaient toujours sur la barrière alpine qu'ils devaient contourner par l'Ouest, avec l'aide des petits réseaux suisses. Ces derniers assuraient la jonction avec le PLM qui contrôlait les accès italiens. Une lutte s'engagea alors entre les chemins de fer français et allemands pour le contrôle des lignes helvétiques qui finirent par passer sous contrôle germanique.

L'ouverture du tunnel du Saint-Gothard, en 1882, dans les Alpes suisses, favorisa nettement Ostende, Anvers, ainsi que les cités hanséatiques, au détriment des ports français du Nord : pour le transit britannique à destination des côtes italiennes, il donnait à la Belgique l'avantage des plus courtes distances et renforçait les lignes rhénanes. En réponse, les milieux portuaires de la Manche 
et de la mer du Nord réclamèrent le creusement d'un tunnel du côté français. Les compagnies renâclèrent devant une telle perspective, en particulier le PLM qui craignait de voir s'effondrer la rentabilité de sa ligne du MontCenis. Les ports étaient, pour leur part, divisés quant à la localisation de cette voie concurrente au Saint-Gothard. Les uns étaient favorables au percement du Mont-Blanc, mais une tendance majoritaire se dessina progressivement en faveur du tunnel du Simplon, dont la réalisation connut de nombreux problèmes, si bien que l'ouverture au trafic fut inaugurée seulement en $1906^{11}$.

De leur côté, les compagnies françaises passèrent progressivement d'un système de concurrence à un système d'entente avec les compagnies étrangères. En 1895, un accord fut conclu pour le partage du trafic des marchandises à grande vitesse en transit entre l'Angleterre et la Suisse. La voie de CalaisBoulogne devait percevoir $60 \%$ des recettes, celles d'Anvers $23 \%$ et celle d'Ostende $17 \%$. Ces pratiques se prolongèrent jusqu'à la veille de la Première Guerre mondiale. En 1909, les tarifs de marchandises destinées à l'Italie firent l'objet d'un accord de partage " pour attribuer au Simplon le trafic qui lui revenait par application de la plus courte distance ${ }^{12}$. Ces ententes s'établirent notamment aux dépens des ports de l'Atlantique qui étaient desservis par d'autres réseaux. Ceci explique que, à la fin du XIX ${ }^{e}$ siècle, les " canalistes " et certains milieux portuaires aient, comme à Nantes, recherché le salut par la construction d'une grande artère fluviale de pénétration vers les marchés de l'Europe moyenne.

La perte de compétitivité des ports français fut jugée suffisamment sérieuse, au tournant des années 1870 , pour que leurs chambres de commerce respectives réclament la constitution de commissions d'enquête auprès des pouvoirs publics. Dès 1878, la Commission des ports maritimes du Conseil supérieur des voies de communication en recommanda une sur les instances des chambres de commerce de Bordeaux, du Havre et de Nantes. De nouveau, Le Havre, appuyé par Dunkerque, formula des plaintes relatives aux tarifs trop élevés que pratiquaient les compagnies de chemins de fer françaises. La chambre de Dunkerque n'hésitait pas à parler « du détournement par la voie d'Anvers du trafic des fers de Suède vers les Ardennes ${ }^{13}$. Ce fut également dans ce contexte anxieux que prit place, en 1882, la grande commission d'enquête, présidée par le député Félix Faure, sous l'égide du Comité consultatif des chemins de fer, qui eut pour objectif précis d'analyser " la situation des

11- VIGARIÉ, 1964, p. 287-288.

12- Cité par CARON, 2005, p. 270.

13- Ministère des Travaux publics, 1883, p. 1. 
ports français de la Manche au point de vue de la concurrence avec les ports étrangers ". Les conclusions de l'enquête liaient la diminution des tarifs de transit, l'amélioration des délais d'acheminement et des communications à l'intérieur du territoire. Ces trois problèmes ne formaient qu'un d'après l'avis des experts.

Les vingt dernières années du siècle se distinguèrent par la multiplication des voyages d'étude et des analyses comparatives confiées aux plus éminents spécialistes des questions portuaires, tels que les ingénieurs Plocq et Laroche ou l'économiste Clément Colson ${ }^{14}$. Cet ébranlement intellectuel eut pour effet de faire évoluer la réflexion de quelques rares dirigeants français. Le plan d'équipement proposé par le ministre des Travaux publics Pierre Baudin, en 1900, était nouveau par son réalisme, qui consistait à concentrer, de façon très nette, les efforts sur quelques établissements. La trop grande dispersion du trafic d'entrée comme de sortie entre les différents ports français était considérée comme une source de faiblesse qui, a fortiori, ne pouvait inciter les compagnies ferroviaires à multiplier leurs lignes et à diminuer leurs tarifs. À l'inverse, les ports réclamèrent constamment l'ouverture de nouvelles liaisons fluviales et ferroviaires pour stimuler leur activité. Une logique inverse était désormais à l'œuvre. Le réalisme de ce plan, qui revenait à promouvoir en particulier la compétitivité des ports septentrionaux du littoral français, fut aussi la cause de son échec relatif. Les crédits finalement accordés étaient bien inférieurs aux besoins initiaux et les procédures imaginées pour raccourcir les délais entre l'adoption de la loi et le début des travaux furent tout simplement rejetées. Les ports qui étaient lésés par le programme Baudin ne furent pas les derniers à en vider l'esprit et le contenu.

Au fond, la même logique discriminante était à l'œuvre dans le projet privé de "Paris port de mer ». Son auteur, l'ingénieur Bouquet de la Grye, avait imaginé que l'on pourrait tracer une carte avec des lignes limitant les zones d'expansion des ports qu'il dénommait « la possibilité commerciale des ports ». Cette carte montrerait comment les marchandises pouvaient aller plus ou moins loin à l'intérieur de l'espace national, en fonction du prix à l'arrivée dans le port et selon le recours aux canaux ou aux chemins de fer. De ses travaux, Bouquet de la Grye tira la conclusion que Paris, qui était déjà le principal nœud de communications terrestres du pays, serait le seul port à pouvoir lutter contre Anvers et Hambourg. Bien évidemment, la plupart des chambres de commerce maritimes s'effrayèrent d'un projet qui prônait une telle vision hiérarchisée de l'espace. Aussi résistèrent-elles fortement à cette

14- PLOCQ et LAROCHE, 1882 ; COLSON et ROUME, 1888. 
logique de concentration et de hiérarchisation des ports et des flux d'échanges à l'échelle supra-nationale. À titre d'exemple, la chambre de commerce de Bordeaux craignit que ce nouveau système économique, qui favorisait l'accès à quelques grands ports maritimes avec redistribution en arrière, ne condamnât définitivement les ports de fond d'estuaire.

La prise en compte des enjeux de la "nodalité » des ports fut bien réelle dans les trente années qui précédèrent la Première Guerre mondiale. Elle risquait cependant d'amplifier un processus de sélection, que le mouvement de modernisation technique du XIx ${ }^{e}$ siècle avait déjà bien enclenché. Elle heurtait trop les principes de la répartition des crédits, de même qu'elle pouvait réduire à néant les efforts menés de longue date par des élites locales soucieuses d'améliorer leur outil.

\section{Conclusion}

"Le port moderne est un vaste carrefour de routes de mer et de routes de terre ", pouvait écrire au début du Xx siècle l'économiste Paul de Rousiers. "Il ne s'ouvre pas seulement vers la mer, il s'ouvre aussi sur l'intérieur des terres; il s'efforce de multiplier les liaisons avec son arrière-pays, car il vit désormais principalement de cet arrière-pays ${ }^{15}$. " La plupart des grands ports français sont devenus des nœuds de communication régionaux, mais rarement au-delà en raison de la faiblesse relative de leurs trafics, en particulier à l'exportation. Seuls quelques établissements, situés dans une relative proximité des grands centres de production et de consommation de l'espace européen, ont pu prétendre un moment au rôle de nœuds de communication de niveau supra-national. Cependant, pour les diverses raisons précédemment évoquées, ni Le Havre ni Dunkerque ou Marseille n'ont pu soutenir la concurrence internationale, étant même menacés dans leur fonction de redistributeurs à l'échelon national. La principale leçon à tirer de l'expérience française réside dans l'incapacité des pouvoirs publics à comprendre les enjeux essentiels du transit international qui fut le support essentiel de la montée en puissance de ports comme Anvers, Rotterdam, Hambourg et Gênes. La politique d'équipement portuaire et d'interconnexion a trop suivi la pente de rivalités étroitement régionales et nationales, jusqu’à adhérer aux prétentions irréalistes de certains établissements, ce qui laisse au total une impression de gâchis.

15- Cité par RONCAYOLO, 1990, p. 43. 


\section{Bibliographie}

CARON, 1997, 2005 : Caron, François, Histoire des chemins de fer en France, tome premier, 1740-1883, Paris, Fayard, 1997 ; tome deuxième, 18831937, Paris, Fayard, 2005.

CLOÎTRE-QUÉRÉ, 1992 : Cloître-Quéré, Marie-Thérèse, Brest et la mer, 1848-1874, Saint-Thonan, Centre de la recherche bretonne et celtique, U.B.O. Brest, 1992.

COLSON et ROUME, 1888 : Colson, Clément et Roume, Ernest, L'Organisation financière des ports maritimes de commerce en Angleterre, Paris, Dunod, 1888.

COLSON, 1898-1903 : Colson, Clément, Transports et tarifs, Paris, J. Rothschild, 1898-1903.

CROGUENNEC, 1999 : Croguennec, Michel, "L'aménagement du port de Rouen de 1800 à 1940 : contraintes, techniques et stratégies ", thèse de doctorat, sous la dir. de Michel Pigenet, Rouen, 1999.

MARNOT, 2000 : Marnot, Bruno, Les Ingénieurs au Parlement sous la Troisième République, Paris, CNRS-Éditions, 2000.

Ministère des Travaux publics, 1883 : Ministère des Travaux publics, Enquête sur la situation des ports français de la Manche au point de vue de la concurrence avec les ports étrangers, Paris, Imprimerie nationale, 1883.

PICARD, 1884 : Picard, Alfred, Les Chemins de fer français, t. 3, Paris, J. Rothschild Éditeur, 1884.

PICARD, 1918 : Picard, Alfred, Les Chemins de fer, Paris, Dunod et Pinat, 1918.

PLOCQ et LAROCHE, 1882 : Plocq, Alexandre et Laroche, Charles, Étude sur les principaux ports de commerce de l'Europe septentrionale publiée par ordre de M. le Ministre des Travaux Publics, Paris, Imprimerie Nationale, 1882.

RONCAYOLO, 1990 : Roncayolo, Marcel, L'Imaginaire de Marseille. Port, ville, pôle, Marseille, CCIP, 1990.

RONCAYOLO, 2000 : Roncayolo, Marcel, «L'aménagement du territoire (XVIII ${ }^{\mathrm{e}}-\mathrm{XX}^{\mathrm{e}}$ siècles) ", in André Burguière, Jacques Revel (dir.), Histoire de la France, tome I, L'Espace français, Paris, Seuil, 2000.

VAUTHIER-VÉZIER, 1997 : Vauthier-Vézier, Anne, " Nantes, le port et la Loire maritime, une histoire culturelle de l'aménagement au XIX siècle ", thèse de doctorat, sous la dir. de J.-Clément Martin, Nantes, 1997. 
VERAGHTERT, 1975 : Veraghtert, Karel, "Anvers et les ports français durant la seconde moitié du XIX ${ }^{e}$ siècle ", in Les Relations franco-belges de 18301934, Actes du colloque de Metz, 15-16 novembre 1974, Metz, Centre de recherches Relations internationales de l'université de Metz, 1975, p. 113.

VIGARIÉ, 1964 : Vigarié, André, Les Grands Ports de commerce de la Seine au Rhin, leur évolution devant l'industrialisation des arrières-pays, Paris, S.A.B.R.I., 1964. 